\title{
Differences in plasma apelin and visfatin levels between patients with type 1 diabetes mellitus and healthy subjects and response after acute hyperglycemia and insulin administration
}

\author{
Kleopatra Alexiadou, ${ }^{1}$ Alexander Kokkinos, ${ }^{1}$ Stavros Liatis, ${ }^{1}$ Despoina Perrea, ${ }^{2}$ \\ Nicholas Katsilambros, ${ }^{1}$ Nicholas Tentolouris ${ }^{1}$
}

${ }^{1}$ First Department of Propaedeutic and Internal Medicine, Athens University Medical School, Laiko General Hospital, ${ }^{2}$ Laboratory of Experimental Surgery and Surgical Research "N.S. Christeas", Athens University Medical School, Athens, Greece

\begin{abstract}
OBJECTIVE. Previous data suggest that apelin and visfatin play a role in metabolism and glucose homeostasis. The aim of the present study was to determine differences in plasma apelin and visfatin concentrations between healthy subjects and patients with type 1 diabetes mellitus and to study the effect of hyperglycemia and insulin administration on their levels in patients with type 1 diabetes mellitus. DESIGN. One hundred patients with T1DM and 52 healthy subjects were examined. Nine patients with type 1 diabetes and 9 controls participated in a further study. In the main study, blood samples were taken after a 12-hour fast. In a further study, an oral glucose tolerance test was performed on two occasions. In session A, at baseline, insulin lispro ( 7 units) was administered subcutaneously to the type 1 diabetic patients, while a placebo injection was administered to controls. In session B, no insulin or placebo was administered. Apelin, visfatin, insulin and glucose levels were measured at baseline and 10, 20, 30, 60, 90, 120,150 and 180 min after glucose consumption. RESULTS AND CONCLUSIONS. Fasting plasma apelin concentrations were higher $(\mathbf{p}<0.001)$, while fasting visfatin levels tended to be lower $(p=0.06)$ in patients with type 1 diabetes in comparison to healthy subjects. In the diabetes group, fasting apelin (but not visfatin) correlated with HDL-C $(p=0.001)$. Apelin and visfatin did not change significantly during the oral glucose tolerance test in either group with or without exogenous insulin administration.
\end{abstract}

Key words: Adipokines, Insulin secretion, Oral glucose tolerance test 


\section{INTRODUCTION}

Apelin and visfatin are two recently described adipokines. Apelin exists in several forms all originating from a common 77 -amino-acid precursor, ${ }^{1}$ but apelin-12 is the most active of these forms. ${ }^{2}$ Its receptor, APJ, is localized in multiple organs and tissues. ${ }^{1,3}$ Data on mice have shown that its synthesis by adipocytes is stimulated by insulin. ${ }^{4}$ The Apelin transcript and protein were identified in adipocytes isolated from mouse and human subcutaneous adipose tissue and its expression increased during adipocyte differentiation. ${ }^{4,5}$

Apelin appears to be involved in the physiological function of numerous organ systems, such as the regulation of blood pressure, cardiac contractility and heart rate. ${ }^{6,7}$ Other studies suggest a potential role for apelin in the regulation of food intake $e^{8-11}$ and water balance. ${ }^{11-13}$ Data have also shown that apelin is associated with angiogenesis, ${ }^{14}$ apoptosis ${ }^{15}$ and inflammation. ${ }^{16}$

Visfatin, a protein previously known as pre-B cell colony-enhancing factor, is a 52-kilodalton cytokine expressed in lymphocytes. Visfatin is involved in the regulation of glucose homeostasis by exerting a hypoglycemic effect through the reduction of glucose release from hepatocytes and the stimulation of glucose utilization in peripheral tissues. ${ }^{5}$ Moreover, it seems to be involved in the regulation of insulin secretion and receptor signaling. ${ }^{17}$ Plasma visfatin concentration correlates strongly with the amount of visceral fat. ${ }^{5}$ Data on mice have shown that plasma levels of this adipokine increase in the obese state and in mice consuming high fat diet. ${ }^{5}$ Mice heterozygous for a targeted mutation in the visfatin gene have modestly higher levels of plasma glucose relative to wild-type littermates. Administration of recombinant visfatin lowers plasma glucose levels in both insulin-resistant and insulin-deficient mice. ${ }^{5}$

Unfortunately, very few data exist on differences in plasma concentrations of apelin and visfatin between subjects with type 1 diabetes mellitus (T1DM), a condition characterized by insulin deficiency, and healthy controls and on the effect of exogenous insulin administration on plasma concentrations of these adipokines. One recent study reported elevated plasma apelin levels in children with T1DM. ${ }^{18}$ Another study showed no differences in plasma apelin levels in women with gestational diabetes mellitus and in women with normal glucose tolerance. ${ }^{19}$

In the present study we looked for potential differences in plasma apelin and visfatin concentrations between healthy controls and subjects with T1DM. Additionally, we studied the effect of a bolus insulin administration on plasma apelin and visfatin levels during an oral glucose tolerance test (OGTT) in patients with T1DM.

\section{SUBJECTS AND METHODOLOGY}

The study was approved by the Ethics Committee of our Hospital. After explanation of the purpose and the procedures of the protocol, all subjects provided written informed consent to participate in the study. A total of 100 subjects with T1DM and 52 healthy controls, similar in terms of age, gender and BMI, were examined. Since BMI may interfere with the levels of apelin and visfatin, the subjects recruited were not obese. The demographics and clinical characteristics of the study subjects are shown in Table 1.

In the morning, after a 12- to 14-hour fast, each subject attended the metabolic unit of our Department. Anthropometric measurements took place at around 8:00 hours. Weight and height were measured in light clothing and BMI was calculated. Waist circumference was measured in the middle between the iliac crest and the lower ribs. Blood was drawn for the determination of plasma glucose, insulin, lipids, apelin and visfatin concentrations.

In addition, 9 non-obese young subjects with well-controlled T1DM ( HbA1c < $7 \%$ ) and 9 healthy subjects, matched in terms of age, gender and BMI, volunteered to participate in a further study in order to examine the effect of a single subcutaneous insulin or placebo administration on plasma concentrations of apelin and visfatin. Subjects with T1DM and controls were studied on two occasions (phases A and B) with a time interval of about 1 week in between in random order according to the permuted block randomization list generated in SPSS. For this purpose, an intravenous catheter was inserted in a superficial forearm vein for blood sampling. In both phases an OGTT was performed. In phase A, before the OGTT 7 units of insulin lispro (approximately 1 unit for every $10 \mathrm{~g}$ of 
Table 1. Clinical and demographic characteristics of the study groups across the two testing days

\begin{tabular}{|c|c|c|c|}
\hline & T1DM & Controls & $\mathrm{P}$ \\
\hline $\mathrm{n}$ & 100 & 52 & \\
\hline Age (years) & $29.2 \pm 0.74$ & $28.6 \pm 0.73$ & 0.43 \\
\hline Body mass index $\left(\mathrm{Kg} / \mathrm{m}^{2}\right)$ & $24.1 \pm 0.33$ & $24.1 \pm 0.59$ & 0.97 \\
\hline Waist $(\mathrm{cm})$ & $81.6 \pm 1.04$ & $83.4 \pm 1.72$ & 0.34 \\
\hline Waist-to-hip ratio & $0.80 \pm 0.01$ & $0.82 \pm 0.01$ & 0.06 \\
\hline Heart rate (beats/min) & $78 \pm 3.02$ & $72 \pm 1.88$ & 0.12 \\
\hline Systolic BP (mm Hg) & $120.13 \pm 13.03$ & $114.56 \pm 14.86$ & 0.03 \\
\hline Diastolic BP (mm Hg) & $76.97 \pm 12.61$ & $68.59 \pm 9.53$ & $<0.001$ \\
\hline Glucose $(\mathrm{mmol} / \mathrm{l})$ & $10.73 \pm 1.51$ & $5.20 \pm 0.24$ & 0.003 \\
\hline $\operatorname{HbA1c}(\%)$ & $7.71 \pm 1.68$ & - & \\
\hline Insulin (pmol/l) & $39.58 \pm 11.38$ & $56.87 \pm 8.34$ & \\
\hline Median value (IQR) & $23.05(19.37-41.80)$ & $63.34(42.21-116.60)$ & $<0.001$ \\
\hline HOMA-IR & - & $1.86 \pm 0.19$ & \\
\hline Median value $(I Q R)$ & - & $2.06(1.45-3.62)$ & \\
\hline Total cholesterol (mmol/l) & $4.48 \pm 0.09$ & $4.56 \pm 0.11$ & 0.55 \\
\hline HDL cholesterol (mmol/l) & $1.46 \pm 0.04$ & $1.17 \pm 0.04$ & $<0.001$ \\
\hline LDL cholesterol (mmol/l) & $3.03 \pm 0.09$ & $3.32 \pm 0.10$ & 0.06 \\
\hline Triglycerides (mmol/l) & $0.77 \pm 0.05$ & $0.98 \pm 0.10$ & \\
\hline Median value (IQR) & $0.61(0.48-0.88)$ & $0.88(0.64-1.10)$ & 0.004 \\
\hline Apelin & $(\mathrm{ng} / \mathrm{ml})$ & $1.89 \pm 0.14$ & $1.25 \pm 0.09$ \\
\hline Median value (IQR) & $1.62(1.15-2.30)$ & $1.26(0.72-1.72)$ & $<0.001$ \\
\hline Visfatin (ng/l) & $17.26 \pm 1.72$ & $22.52 \pm 3.30$ & \\
\hline Median value (IQR) & $15.30(10.09-20.04)$ & $17.52(13.39-23.51)$ & 0.06 \\
\hline
\end{tabular}

Data are shown as mean \pm SEM, unless otherwise indicated. P values correspond to independent samples T test. BP: blood pressure; IQR: interquartile range.

glucose consumed) was administered subcutaneously in the patients with T1DM, while an equal volume of water for injection (placebo) was administered in the control group. In phase B, no insulin or placebo was administered in either patients with T1DM or controls. Plasma levels of glucose, insulin, apelin and visfatin were measured at baseline and 10, 20, 30, 60, 90, 120, 150 and $180 \mathrm{~min}$ after glucose consumption.

Lipids and glucose levels were measured on an automatic enzymatic analyzer. Plasma insulin concentrations were measured by radioimmunoassay (Biosure, Brussels, Belgium; intra- and inter-assay coefficient of variation $<4 \%$ ), while plasma apelin and visfatin levels were determined by ELISA (Phoenix Pharmaceuticals, California, CA, USA; intra- and inter-assay coefficient of variation $<5 \%$ ).
Statistical analysis was performed using the SPSS program (version 12.0; SPSS, Chicago, IL, USA). Values are reported as means \pm SEM. For categorical variables, differences between the controls and subjects with T1DM were compared with the chisquare test. For continuous variables with normal distribution of the values, differences between the two groups were tested using the Student's t-test, while the Mann-Whitney U test was used for triglycerides, insulin, fasting apelin and visfatin levels because the data were skewed. Bivariate correlations were tested using the Spearman's correlation coefficient after Bonferroni correction. ANOVA for repeated measurements was performed to test the timing effect of the studied parameters in the two phases of the study. The same analysis was used to examine for differences during the experiment in plasma concen- 
trations of the study parameters after adjustment for the status of the participants (T1DM/healthy controls) (between-subjects effects). The Greenhouse-Geisser adjustment was used when the sphericity assumptions were not fulfilled. Postprandial responses of the serial measurements of plasma apelin and visfatin were summarized, calculating AUCs using the trapezoid rule. ${ }^{20} \mathrm{P}$ values $<0.05$ (two-tailed significance) were considered statistically significant.

\section{RESULTS}

As expected, fasting glucose levels were higher and insulin levels lower in subjects with T1DM in comparison with the controls. Age, gender, BMI, waist circumference, blood pressure did not differ significantly between the two groups; however, LDL cholesterol and triglycerides levels were lower and HDL cholesterol concentrations were higher in subjects with T1DM than controls (Table 1).

Fasting plasma apelin concentrations were significantly higher $(\mathrm{p}<0.001)$, while fasting visfatin levels tended to be lower in subjects with T1DM than in controls $(p=0.06)$. In the diabetes group, fasting apelin concentrations correlated significantly (after Bonferroni correction) with HDL-C ( $\mathrm{r}=0.38$, $\mathrm{p}=0.001$ ), while no significant correlation was found with age, BMI, waist circumference, blood pressure, duration of diabetes, $\mathrm{HbA} 1 \mathrm{c}$, insulin, visfatin and the other plasma lipids. In the control group, no significant correlations were found between fasting apelin levels and the above variables. In addition, no significant correlations were found between plasma visfatin levels and the above parameters in either the diabetes or the control group.

Plasma apelin and visfatin levels did not change significantly during phase A of the experiment in either the group of patients with T1DM or the controls (ANOVA for repeated measurements, $p$ value for the effect of time $>0.05$ ) and there was no significant difference between the two groups (ANOVA for repeated measurements, time by group interaction $>0.05$ ) (Figure 1a and 1b). The same was valid for phase B of the study (Figures 1a and 1b). The overall apelin response, expressed as AUC, was not different between T1DM and controls during either session $\mathrm{A}$ $(0.67 \pm 0.16$ vs. $0.73 \pm 0.19 \mathrm{ng} \cdot \mathrm{min} / \mathrm{mL}, \mathrm{p}=0.80)$ or

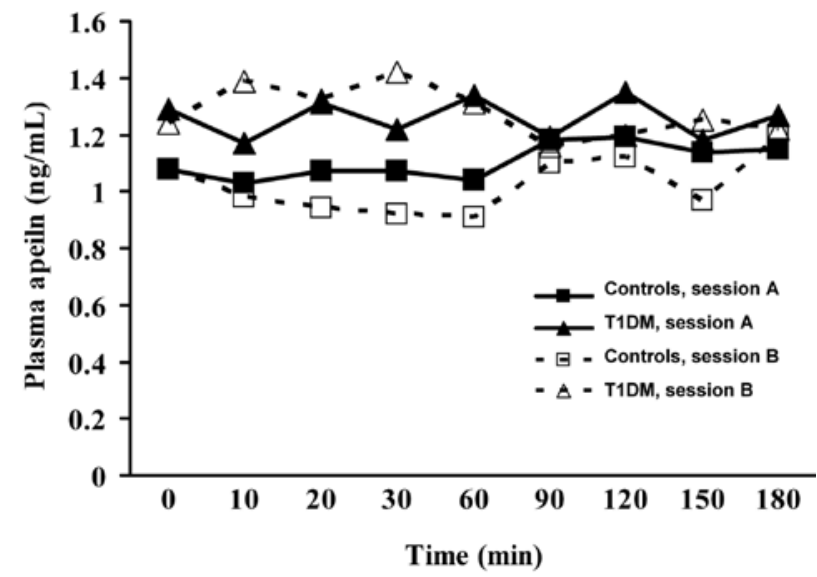

Figure 1a. Plasma apelin levels in type 1 diabetic patients and healthy subjects during an OGTT with (session A) or without (session B) insulin administration.

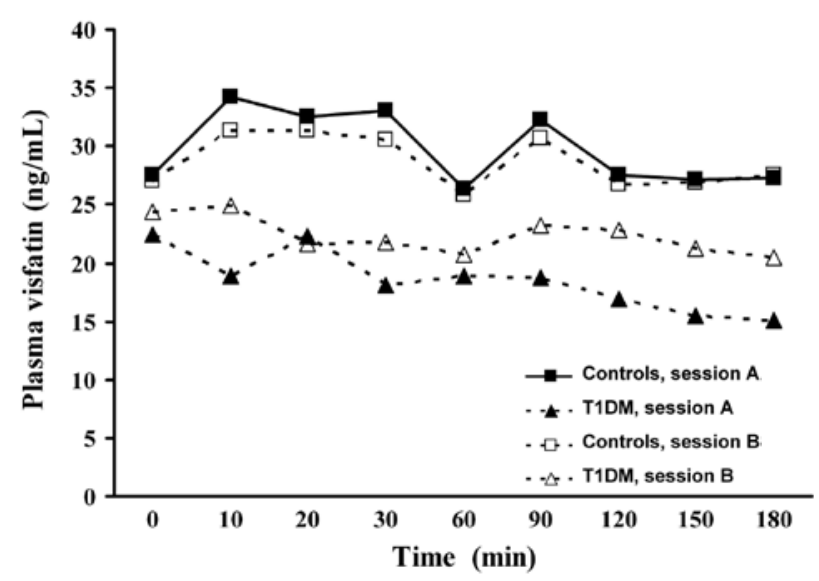

Figure 1b. Plasma visfatin levels in type 1 diabetic patients and healthy subjects during an OGTT with (session A) or without (session B) insulin administration.

B $(0.72 \pm 0.15$ vs. $0.79 \pm 0.14 \mathrm{ng} \cdot \min / \mathrm{mL}, \mathrm{p}=0.74)$. The overall visfatin response, expressed as AUC, was not different between T1DM and controls in either session A $(24.65 \pm 12.15$ vs. $75.72 \pm 31.51 \mathrm{ng} \cdot \mathrm{min} /$ $\mathrm{mL}, \mathrm{p}=0.18)$ or $\mathrm{B}(31.08 \pm 15.35 \mathrm{vs} .45 .86 \pm 23.64 \mathrm{ng}$ $\cdot \min / \mathrm{mL}, \mathrm{p}=0.61)$.

Plasma glucose levels increased significantly during both phases of the study in both controls and patients with T1DM ( $\mathrm{P}$ values for the effect of time in both groups $<0.001$ ) and the increase was higher in the patients with T1DM ( $\mathrm{p}$ value for the time by group interaction in phase $\mathrm{A}=0.02$ and in phase $\mathrm{B}$ $<0.001)$. Plasma insulin levels increased significantly 
in the controls during phase A and phase $\mathrm{B}$ ( $\mathrm{p}$ value for the effect of time $<0.001$ for both phases) but they did not change in the patients with T1DM. The increase in plasma insulin levels were significantly higher in the controls in comparison with the patients with T1DM ( $\mathrm{p}$ value for the time by group interaction $<0.001$ for both phases).

\section{DISCUSSION}

In the present study we showed that fasting plasma apelin concentrations were higher in patients with T1DM in comparison with healthy subjects. The lack of endogenous insulin synthesis and secretion in patients with T1DM is associated with high circulating apelin levels. Therefore, our data suggest that insulin may affect the expression of apelin in adults.

Most of the studies on mice and humans have shown that apelin expression and secretion increases under conditions of chronic hyperinsulinemia such as the obese state or type 2 diabetes, implying that insulin is involved in the regulation of circulating apelin levels. ${ }^{3,21-23}$ Only two studies found low plasma apelin levels in newly diagnosed type 2 diabetic patients. ${ }^{24,25}$ Some animal studies have linked apelin to insulin resistance and considered this adipokine as an important factor for the maintenance of insulin sensitivity and the stimulation of glucose utilisation by the peripheral tissues. ${ }^{26-28}$ The decrease in apelin levels after weight $\operatorname{loss}^{29}$ supports the hypothesis of the interaction between apelin and insulin resistance. The increased levels of apelin observed in obesity could be a mechanism to counterpace insulin resistance. Another study in mice revealed the existence of the apj receptor in the pancreatic islets and concluded that apelin inhibits the glucose-stimulated insulin secretion. ${ }^{30}$ Thus, one could speculate that "hyperapelinemia" is a sort of negative feedback mechanism which combats hyperinsulinemia in obesity or type 2 diabetes. On the other hand, streptozotocin-treated insulin-deficient mice had a large decrease in apelin expression $^{3}$ but there is no evidence of apelin levels in adults without insulin secretion. There is only one recent study where circulating apelin levels were found elevated in children with T1DM compared to controls. ${ }^{18}$ The higher levels of apelin found in our patients with T1DM could be an attempt to compensate for the lack of insulin in the same way that raised apelin levels in obesity or type 2 diabetes could possibly try to overcome insulin resistance and substitute the relative "lack" of insulin. We showed that fasting apelin levels correlated positively with HDL cholesterol. A negative correlation between fasting apelin levels and serum triglycerides has been reported in animals. ${ }^{26}$

In our study, neither apelin nor insulin levels increased during OGTT in the diabetic group. In addition, in the control group the normal insulin secretion was not accompanied by an increase in apelin secretion. These findings suggest that apelin levels do not change acutely and they are not affected by blood glucose levels since they remained unchanged under either normoglycemic or hyperglycemic conditions. This finding agrees with data on mice showing that high glucose concentration itself did not affect apelin m-RNA levels. ${ }^{4} \mathrm{~A}$ single insulin injection increased apelin m-RNA levels in adipocytes isolated from mice but did not affect apelin secretion. The effect of insulin on apelin expression and secretion was dose-dependent. ${ }^{4}$ In our study apelin levels were lower than those of other peptides thought to function as circulating hormones and they were comparable to the levels of locally acting mediators, implying a potent paracrine action of apelin. Isolation of apelin m-RNA of adipocytes from both controls and patients with T1DM across the two testing days could have led to interesting findings.

Regarding visfatin, in our study fasting visfatin levels tended to be lower in patients with T1DM compared to healthy subjects, implying a potential effect of insulin deficiency on visfatin secretion.

Visfatin binds to and activates the insulin receptor in a way distinct from insulin. Several studies demonstrated that plasma visfatin levels correlate with obesity, visceral fat mass, type 2 diabetes and the presence of the metabolic syndrome..$^{31-33}$ Other studies, however, did not confirm an association of visfatin with visceral adipose tissue or parameters of insulin sensitivity in humans and rodents. ${ }^{34-36}$ In our study no association was found between visfatin levels and metabolic parameters in either the type 1 diabetic or the non-diabetic group.

Visfatin levels were found to be higher in newly 
diagnosed patients with type 2 diabetes mellitus ${ }^{37}$ and lower in patients with long-term disease treated with antidiabetic agents compared to controls. ${ }^{21}$ This could confirm the hypothesis that hyperinsulinemia as a consequence of insulin resistance leads to elevated visfatin levels, while insulinopenia as a result of longstanding type 2 diabetes is followed by a decrease in visfatin levels. The result of our study is consistent with one previous report on type 1 diabetes. ${ }^{38}$ Another study reported increased circulating visfatin levels in patients with T1DM, seeking to associate them with the deterioration of $\beta$-cell function. ${ }^{39}$ On the other hand, in vitro and in vivo animal experiments showed a potent regulatory role of visfatin in insulin secretion and signalling in pancreatic islets. ${ }^{17,40}$ Visfatin deficiency or inhibition resulted in decreased glucose-stimulated insulin secretion, ${ }^{40}$ whereas pancreatic $\beta$-cell incubation with visfatin at low glucose increased insulin secretion. ${ }^{17}$ Therefore, one may hypothesize that visfatin deficiency coexists with insulin deficiency in T1DM or even contributes to it. Further research is needed in order to determine if the association between circulating visfatin and insulin levels in T1DM denotes causality or simply coexistence.

The second part of the present study examined the potential acute changes in visfatin levels under hyperglycemic conditions with or without insulin secretion during an OGTT. In subjects with type 1 diabetes, insulin administration did not affect visfatin secretion. During placebo injection the visfatin response caused by the hyperglycemia was similar between controls and type 1 diabetes subjects. These findings are in discordance with previous studies demonstrating that basal visfatin release is enhanced by hyperglycemia and counterregulated by exogenous hyperinsulinemia in healthy humans. ${ }^{41}$

The main limitation of our study is the small number of subjects participating in the interventional part of the experiment. Clearly, our study is too underpowered to detect a potential differential response of plasma apelin and visfatin levels in the controls and in the subjects with T1DM during the experiment; therefore, a type II statistical error cannot be excluded. Future studies with a larger sample of participants could elucidate and provide further support to our findings.
In conclusion, circulating fasting apelin levels are higher and visfatin levels tend to be lower in subjects with T1DM compared to healthy controls and do not change significantly during acute hyperglycemia or exogenous insulin administration. The potential association of apelin and visfatin with insulin secretion and action may reveal new pathways in the pathogenesis of type 1 diabetes. Understanding the physiological role of these adipokines in glucose homeostasis and the mechanisms underlying their action is a challenge that could lead to new therapeutic targets.

\section{Conflict of interest: None}

\section{REFERENCES}

1. Lee DK, Cheng R, Nguyen T, et al, 2000 Characterization of apelin, the ligand for the APJ receptor. J Neurochem 74: $34-41$.

2. Tatemoto K, Takayama K, Zou MX, et al, 2001 The novel peptide apelin lowers blood pressure via a nitric oxide-dependent mechanism. Regul Pept 99: 87-92.

3. Kleinz MJ, Davenport AP, 2005 Emerging roles of apelin in biology and medicine. Pharmacol Ther 107: 198-211.

4. Boucher J, Masri B, Daviaud D, et al, 2005 Apelin, a newly identified adipokine up-regulated by insulin and obesity. Endocrinology 146: 1764-1771.

5. Bełtowski J, 2006 Apelin and visfatin: unique "beneficial" adipokines upregulated in obesity? Med Sci Monit 12: RA112-119.

6. Szokodi I, Tavi P, Földes G, et al, 2002 Apelin, the novel endogenous ligand of the orphan receptor APJ, regulates cardiac contractility. Circ Res 91: 434-440.

7. Ashley EA, Powers J, Chen M, et al, 2005 The endogenous peptide apelin potently improves cardiac contractility and reduces cardiac loading in vivo. Cardiovasc Res 65: 73-82.

8. Sunter D, Hewson AK, Dickson SL, 2003 Intracerebroventricular injection of apelin-13 reduces food intake in the rat. Neurosci Lett 353: 1-4.

9. O’Shea M, Hansen MJ, Tatemoto K, Morris MJ, 2003 Inhibitory effect of apelin-12 on nocturnal food intake in the rat. Nutr Neurosci 6: 163-167.

10. Jászberényi M, Bujdosó E, Telegdy G, 2004 Behavioral, neuroendocrine and thermoregulatory actions of apelin-13. Neuroscience 129: 811-816.

11. Taheri S, Murphy K, Cohen M, et al, 2002 The effects of centrally administered apelin-13 on food intake, water intake and pituitary hormone release in rats. Biochem Biophys Res Commun 291: 1208-1212.

12. Reaux A, De Mota N, Skultetyova I, et al, 2001 Physiological role of a novel neuropeptide, apelin, and its receptor in the rat brain. J Neurochem 77: 1085-1096. 
13. Reaux-Le Goazigo A, Morinville A, Burlet A, LlorensCortes C, Beaudet A, 2004 Dehydration-induced crossregulation of apelin and vasopressin immunoreactivity levels in magnocellular hypothalamic neurons. Endocrinology 145: 4392-4400.

14. Kasai A, Shintani N, Oda M, et al, 2004 Apelin is a novel angiogenic factor in retinal endothelial cells. Biochem Biophys Res Commun 325: 395-400.

15. Xie H, Yuan LQ, Luo XH, et al, 2007 Apelin suppresses apoptosis of human osteoblasts. Apoptosis 12: 247-254.

16. Daviaud D, Boucher J, Gesta S, et al, 2006 TNFalpha up-regulates apelin expression in human and mouse adipose tissue. FASEB J 20: 1528-1530.

17. Brown JE, Onyango DJ, Ramanjaneya M, et al, 2010 Visfatin regulates insulin secretion, insulin receptor signalling and mRNA expression of diabetes-related genes in mouse pancreatic beta-cells. J Mol Endocrinol 44: 171-178.

18. Meral C, Tascilar E, Karademir F, et al, 2010 Elevated plasma levels of apelin in children with type 1 diabetes mellitus. J Pediatr Endocrinol Metab 23: 497-502.

19. Telejko B, Kuzmicki M, Wawrusiewicz-Kurylonek N, et al, 2010 Plasma apelin levels and apelin/APJ mRNA expression in patients with gestational diabetes mellitus. Diabetes Res Clin Pract 87: 176-183.

20. Matthews JN, Altman DG, Campbell MJ, Royston P, 1990 Analysis of serial measurements in medical research. BMJ 300: 230-235.

21. Li L, Yang G, Li Q, et al, 2006 Changes and relations of circulating visfatin, apelin, and resistin levels in normal, impaired glucose tolerance, and type 2 diabetic subjects. Exp Clin Endocrinol Diabetes 114: 544-548.

22. Heinonen MV, Purhonen AK, Miettinen P, et al, 2005 Apelin, orexin-A and leptin plasma levels in morbid obesity and effect of gastric banding. Regul Pept 130: 7-13.

23. Soriguer F, Garrido-Sanchez L, Garcia-Serrano S, et al, 2009 Apelin levels are increased in morbidly obese subjects with type 2 diabetes mellitus. Obes Surg 19: 1574-1580.

24. Erdem G, Dogru T, Tasci I, Sonmez A, Tapan S, 2008 Low plasma apelin levels in newly diagnosed type 2 diabetes mellitus. Exp Clin Endocrinol Diabetes 116: 289-292.

25. Zhang Y, Shen C, Li X, et al, 2009 Low plasma apelin in newly diagnosed type 2 diabetes in Chinese people. Diabetes Care 32: e150.

26. Higuchi K, Masaki T, Gotoh K, et al, 2007 Apelin, an APJ receptor ligand, regulates body adiposity and favors the messenger ribonucleic acid expression of uncoupling proteins in mice. Endocrinology 148: 2690-2697.

27. Dray C, Knauf C, Daviaud D, et al, 2008 Apelin stimulates glucose utilization in normal and obese insulin- -resistant mice. Cell Metab 8: 437-445.

28. Yue $\mathrm{P}$, Jin H, Aillaud M, et al, 2010 Apelin is necessary for the maintenance of insulin sensitivity. Am J Physiol Endocrinol Metab 298: E59-67.

29. Castan-Laurell I, Vítkova M, Daviaud D, et al, 2008 Effect of hypocaloric diet-induced weight loss in obese women on plasma apelin and adipose tissue expression of apelin and APJ. Eur J Endocrinol 158: 905-910.

30. Sörhede Winzell M, Magnusson C, Ahrén B, 2005 The apj receptor is expressed in pancreatic islets and its ligand, apelin, inhibits insulin secretion in mice. Regul Pept 131: 12-17.

31. Chen MP, Chung FM, Chang DM, et al, 2006 Elevated plasma level of visfatin/pre-B cell colony-enhancing factor in patients with type 2 diabetes mellitus. J Clin Endocrinol Metab 91: 295-299.

32. Haider DG, Schindler K, Schaller G, Prager G, Wolzt M, Ludvik B, 2006 Increased plasma visfatin concentrations in morbidly obese subjects are reduced after gastric banding. J Clin Endocrinol Metab 91: 1578-1581.

33. Filippatos TD, Derdemezis CS, Gazi IF, et al, 2008 Increased plasma visfatin levels in subjects with the metabolic syndrome. Eur J Clin Invest 38: 71-72.

34. Berndt J, Klöting N, Kralisch S, et al, 2005 Plasma visfatin concentrations and fat depot-specific mRNA expression in humans. Diabetes 54: 2911-2916.

35. Pagano C, Pilon C, Olivieri M, et al, 2006 Reduced plasma visfatin/pre-B cell colony-enhancing factor in obesity is not related to insulin resistance in humans. $\mathrm{J}$ Clin Endocrinol Metab 91: 3165-3170.

36. Oki K, Yamane K, Kamei N, Nojima H, Kohno N, 2007 Circulating visfatin level is correlated with inflammation, but not with insulin resistance. Clin Endocrinol (Oxf) 67: 796-800.

37. Dogru T, Sonmez A, Tasci I, et al, 2007 Plasma visfatin levels in patients with newly diagnosed and untreated type 2 diabetes mellitus and impaired glucose tolerance. Diabetes Res Clin Pract 76: 24-29.

38. Toruner F, Altinova AE, Bukan N, et al, 2009 Plasma visfatin concentrations in subjects with type 1 diabetes mellitus. Horm Res 72: 33-37.

39. López-Bermejo A, Chico-Julià B, Fernàndez-Balsells $M$, et al, 2006 Serum visfatin increases with progressive beta-cell deterioration. Diabetes 55: 2871-2875.

40. Revollo JR, Körner A, Mills KF, et al, 2007 Nampt/ $\mathrm{PBEF} /$ Visfatin regulates insulin secretion in beta cells as a systemic NAD biosynthetic enzyme. Cell Metab 6: 363-375.

41. Haider DG, Schaller G, Kapiotis S, Maier C, Luger A, Wolzt M, 2006 The release of the adipocytokine visfatin is regulated by glucose and insulin. Diabetologia 49: 1909. 